\title{
Literature Review of Syntactic Priming Experiment Methods and Bilingual Speech Production Models
}

\author{
Rouhua Wang \\ Foreign Language College \\ Northeast Normal University \\ Changchun, China 130024 \\ Changchun Institute of Technology \\ Changchun, China 130022
}

\author{
Yongbing Liu* \\ Foreign Language College \\ Northeast Normal University \\ Changchun, China 130024 \\ *Corresponding Author
}

\begin{abstract}
Syntactic priming refers to the tendency of people to repeatedly use sentence structures that have just been processed in speech production. Since Bock's first research, syntactic priming has become an important field for the study of syntactic representation and sentence generation mechanism from the perspective of psycholinguistics, and has received more and more attention. The application of syntactic priming experiment method provides a window for us to study some of the internal mechanisms of human syntactic representation and language processing, and also provides a scientific basis for revealing the internal mechanism of syntactic representation. This article introduces the experimental paradigms of foreign syntactic priming and its experimental methods, including Bock's research paradigm, the syntactic reconstruction hypothesis of Potter and Lombordi, and the lexical syntax layer theory of Pichering and Branigan. The experiments have all achieved a priming effect. These theories explain the process of syntactic representation and sentence production in detail and help to reveal the mechanism of sentence comprehension. Second language acquisition is a very complex psychological process involving many relevant factors. Since the birth of this discipline, scholars have explored cross-sectional and in-depth understanding of second language acquisition from various fields such as applied linguistics, psycholinguistics, neurolinguistics, and sociolinguistics, and have proposed various Second language acquisition theories. Based on Levelt's mother tongue output model, this article briefly introduces De Bot and Kormos et al.'s bilingual speech production models, explains the application and development of the theory, and finally points out the deficiency of the bilingual speech production models.
\end{abstract}

Keywords-syntactic priming experiment methods; bilingual speech production models; syntactic representation

\section{INTRODUCTION}

People tend to repeat their use of sentence types or structures when they speak, a phenomenon called syntactic priming (Pickering, M.J, Branigan, H.P.1999). Some people have made this phenomenon syntactic persistence or structural priming. For example, a subject has just contacted an active sentence, such as "The teacher suggested a new plan", when the subject asked to describe in the picture, the subjects tend to use active sentences, such as "A car is pushing an old woman". However, if the previous contact was a passive sentence, such as "A new plan has been suggested by the teacher", the subject would tend to use a passive sentence when describing the same picture, such as "An old man is being pushed by the car". Bock explained that this effect stems from the priming of the syntactic structure.

Syntactic priming has become a method of studying the syntactic representation and generative mechanism from the perspective of psycholinguistics. It has received more and more attention and has become an important research field. It is also used by psycholinguists to study the psychological representation of syntax, which is ultimately used to explain language processing mechanisms and to explain the relationship between language understanding and language production (Yang Jie, ZhangYaxu.2007) In 1986, Bock first studied the phenomenon of syntactic priming. Syntactic priming studies to date have involved many aspects. There are also a lot of research methods used, including sentence completion method, sentence recall, picture description method, alliance script technology. These studies provide a method for in-depth exploration of the syntactic process, confirm the importance of the priming phenomenon in the process of the production of relevant sentences, and provide possibilities for the study of different aspects of language cognitive science. They have become syntactic representations and generating mechanisms from the perspective of psycholinguistics.

The application of syntactic priming experiment method provides a window for us to study some of the internal mechanisms of human syntactic representation and language processing, and also provides a scientific basis for revealing the internal mechanism of syntactic representation. This article introduces the experimental paradigms of foreign syntactic priming and its experimental methods, including Bock (1986a)'s research paradigm, Potter's and Lombardi's syntactic reconstruction hypothesis, and Pichering's and Braniga's vocabulary syntax layer theory. The three different experimental paradigms use a starting sentence that allows participants to accept a specific structure, resulting in many possible target sentences. Although the theoretical explanations of the various scholars are inconsistent, all 
experiments have obtained the priming effect. It also proves that the syntax priming reflects the problem of linguistic representation and that vocabulary, speech, or communication strategies are not enough to explain this phenomenon.

Second language acquisition research began in the 1970s. In January 1975, during the Sixth Annual Meeting of Applied Linguistics at the University of Michigan, a second language acquisition research conference was held. The second language acquisition study was born as a new discipline. The interdisciplinary nature of the discipline and its own complexity determine that it is constantly updated and dynamic. In the 1970s, through the study of error analysis and inter-language, people revealed the process of second language acquisition and made certain the nature of the second language acquisition language system. Exploration aims to discover the stage of development of language acquisition. With the gradual deepening of the study of second language acquisition, the transitional language research has added content of transitional variables caused by factors such as individual, pragmatic environment and communicative tasks. In the $1980 \mathrm{~s}$, the focus of research on second language acquisition shifted to the performance analysis of learners using cognitive psychology and generative linguistics, and then turned to input words for learners. (in put) conducts discourse analysis. Levelt (1989) first proposed a speech output model for the mother tongue, and later Levelt (1999) modified the previously proposed native language output model. He believes that language output is modular, that is, certain processing components in the system are relatively self-sufficient in function, they have their own specific inputs, and are processed independently of other components. The model is mainly a language-generated process model of vocabularydriven modules. It laid the foundation of psycholinguistics for us to study the second language output. Based on Levelt (1999)'s native language output model, De Bot proposes a bilingual language output model. The author made a brief introduction to the origin of the bilingual speech production models, the main content, the application and development of the bilingual speech production models, and put forward his views on the inadequacies.

\section{SyNTACTIC PRIMING EXPERIMENT METHODS}

\section{A. Bock's Experimental Paradigm}

Bock first observed syntactic priming effects in the laboratory. The experiment is based on the reality of the flexibility of the human language sentence structure, that is, the same concept can be expressed in two different sentence patterns. The experiment used four kinds of priming sentences: active sentences and passive sentences of transitive verb structures, and PO structures and DO structure sentences. In Bock's first experiment, the subjects knew nothing about the purpose of the experiment. After repeated repetitions of the start-up sentence, the subject is required to describe the task picture that has no relation to the start-up sentence. The description can use the transitive verb structure or the alternating structure with the lattice.
The experimental results show that the test subject to the influence of the previously repeated start-up sentence is more inclined to use the special syntactic structure in the previous start-up sentence. If you have just heard a passive form of priming sentence, the subject will say a passive sentence, such as "The church was struck by lightning". Also when you hear the activation sentence of the active sentence, the subject will speak an active sentence. Such as "Lightning struck the church". On the other hand, under the influence of the priming structure of the prepositional structure, the subject is more likely to speak a prepositional sentence, such as "The girl hand a hair brush to her mother". Similarly, in the double-object priming sentence, the next test also produced a double-object sentence, such as "The girl hand her mother a hairbrush". (Bock,1986b) In further experiments, Bock used picture description to prove that the vocabulary, the subject and the rhythm consistency between the starting sentence and the target sentence cannot be used to explain the syntactic priming phenomenon, such as having a similar rhythm. A structure like "Susan brought a book to study." could not start the sentence "The girl handed a paint brush to the man."

In another experiment of Bock (1989), Bock found that the prepositions in the start-up sentence and the target sentence are different, and the PO structure start-up sentence can also start the PO structure target sentence, such as the start-up sentence "The secretary baked a cake for her. Boss". There is a start effect on the target sentence "The girl handed a paint brush to the man." The PO structure start sentence representing the place may start a target sentence of a PO structure that does not represent the place, such as the start phrase "The wealthy widow drove her Mercedes to the church." and the goal of "The girl handed a paintbrush to the man." Sentences have a start effect. Including a start phrase of a prepositional phrase that represents a place, such as "The foreigner was loitering by the broken traffic light." a passive sentence containing a by phrase representing the agent can be started.

Bock starts with the activation system interpretation syntax. They believe that the start-up effect is due to the fact that the specific syntactic structure in the language processing process is active, thereby increasing the frequency of selection for repeated use of the syntactic structure. For the first time, Bock's research clearly indicates that the syntactic priming effect is independent of the surface relationships of consecutive sentences and that it is independent of vocabulary, stemming and word meaning levels, and is not affected by the memory of the surface elements of previous sentences. The priming effect is neither determined by the discourse component nor by the communicative purpose. The start-up effect reflects people's abstract recognition of grammatical structure.

\section{B. Potter-lombardi Instantaneous Memory Paradigm}

After Bock's first study, more psychologists began to study the syntactic priming and its importance to language production mechanisms. Potter and Lombardi's sentence recalling experimental paradigmatic research is of great value. Their results have the same syntactic priming effect 
as Bock. The Bock experiment focuses on the activation of special syntactic structures, while Potter and Lombardi focus on the superficial syntax of recall sentences (Potter, M.C, Lombardi, L.1998).

In previous studies, psychology students "module" and "step-by-step recall" explained the ability of people to recall sentences because they thought that recall was a reconstruction of the meaning of sentences in memory and used recently activated words to re-express the full meaning. Potter and Lombardi believe that recalling the meaning of a sentence is as important as the priming of a syntactic sentence.

In Potter's and Lombardi's experiments, there were two types of alternating sentences: sentence and sentence. The experimental results show that the syntactic experiment effects are effective in the independent sentence and the sentence composed of two small clauses. Similar syntactic structures increase the correlation between recall sentences (degree of association is 15\%). The priming effect is effective at recalling priming sentences and receiving recollections. In their three articles, a hypothesis was put forward. The sentence recalling surface syntax does not directly represent memory, but it is reconstructed by the normal mechanism of sentence generation, in which the verb determines the sentence structure (Bock, 1989).

Recalling the correlation between sentences does not lead to sentence surface component memory, but instead of sentence comprehension, the use of activated vocabulary items restructures the syntactic structure. Therefore, from the point of view of syntactic priming, Potter and Lombardi developed the "short-term memory" theory of instantaneous sentence memory, thereby focusing only on the conceptual representation of sentence meaning.

\section{Pickering and Branigan Sentence Completion Paradigm}

Pickering and Branigan explained syntactic priming from the lexical level in 1998. Pickering and Branigan define the lexical syntax layer as three kinds of syntactic information: category information (verbs, nouns, adjectives), characteristic information (numbers, personal names, tenses), combined information, they think that words and language units can form new language expressions. . Pickering and Branigan use a sentence completion task to explain the syntactic priming in written language output. The initiating sentence and the target sentence can be either a prepositional (PO) sentence structure or a double-object (DO) sentence structure. Experiments have shown that it is easier to start sentences with the same syntactic structure when completing written sentences and spoken language picture description tasks. Generated target sentences, and they also found that differences in tense, posture, verbs and numbers in the initiating and target sentences did not affect the priming. They further indicated that they were initiated either in the production of written sentences or in the production of oral sentences. Consistent verbs in the sentence and target sentence enhance the priming effect (Pickering, M.J, Branigan, H.P.1998).
Different from other people's experiments, their interpretation of syntactic priming is based on the theoretical basis of the lexical and syntactic layer. They believe that the generation of words includes the activation of related nodes of the lexical layer and verb entries linked to the combination nodes. Although these activations will decline, they still dominate the subsequent sentences. Experiments by Pickering and Branigan show that syntactic priming can be driven by verbs. For example, the verb give has at least two nodes, indicating the combination of "NP NP" and "NP PP." The sentences of the verb and prepositional (PO) phrase structure include at least one combination node activation, while the verb and doubleobject (DO) phrase structure include the activation of another combination node. The girl handed a hairbrush to her mouth, the word handed activates the relevant entry of the hand. The past tense is the characterization node of this sentence. It is also a combination node of NP and PP. It connects one term to another. Therefore, in the target sentence, the NP PP combination node is easier to reuse and activate than the NP combination node. In other words, in the second output of the sentence, the NP PP node is easier to activate and easier to generate.

They also concluded that the different forms of special verbs do not affect the priming effect, because these verbs are in the same term, and no matter how the verbs are changed in the initiating and target sentences, but the priming effect is not weakened. This shows that the syntactic activation is the syntactic representation of the entry layer.

\section{The BILINGUAL SPEECH PRODUCTION MOdELS}

\section{A. The Origin of the Bilingual Speech Production Models}

Based on Levelt's (1989) mother language output model, De Bot proposed a bilingual speech production models. Levelt believes that information generation is initiated by concepts. In the conceptualizer, the speaker generates communicative intent and performs information coding processing in two stages of macroplanning and mircroplanning to convert it into pre-verbal information. The preverbal information is generated in the next processing unit composer by calling the appropriate term in the mental lexicon, applying the grammar and the voice rule to generate an internal speech plan (speech plan). For the psychological lexicon, Levelt divides it into lexical information composed of lexical semantic information and syntactic information (lemma) and morphological form and form information composed of voice information. The final articulator translates the speech plan into actual discourse by issuing instructions to the neuromuscular system. A major feature of the model is that it takes into account the role of a speech understanding system associated with verbal listening, which monitors the speech plan and the actual spoken words, providing timely feedback. In this way, verbal output and verbal understanding are organically linked together, which strongly illustrates the selfmonitoring function of speech. In general, the Levelt (1989) model is a parallel and incremental model. In the process of 
speech production, the various aspects of speech processing are not sequentially followed, but even if the current stage has not yet been processed. Complete, the next phase of processing has begun. Moreover, language processing at a lower level is more automated than at a higher level.

\section{B. Views and Assumptions of the Bilingual Speech Production Models}

De Bot inherits Levelt's point of view. He believes that the bilingual speech production models are a default system. When a person uses the system, he always uses only one of the languages. Thus, the bilingual speech production models are not essentially different from the monolingual output model. De Bot assumes a shared concept modeler that is responsible for the choice of language and forms information expressed in the chosen language, following the theoretical assumptions of the bilingual vocabulary system, using the subsystem hypothesis of the thesaurus in the bilingual speech production models. A thesaurus contains two language-specific sub-words, two discourse generators to accommodate two thesaurus, each of which contains two language-specific morphological syntax information. They also proposed an extension system for the pronunciation device, which believes that bilinguals usually have a "foreign accent" when speaking a second language because the two languages share a single pronunciation device. This model is essentially consistent with the native language output model.

The bilingual speech production models are based on the improvement of the native language output model. There are three main aspects: First, De Bot believes that for bilinguals, the macro plan is shared by all languages from the communicative intent to the translation of pre-verbal information., and micro-plans need to be set according to different requirements of different languages. Second, De Bot endorses the "subset hypothesis" in bilingual storage, arguing that different languages share a mental lexicon, and the connections between members are strengthened by repeated co-occurrence. This means that the connections between the terms in the same language are stronger than the connections between the different language terms, so the terms of the same language form a subset, and the words can be extracted separately; but under certain conditions, different The connection between the language components can reach the same intensity, so as to realize the random switching between different languages. Therefore, "a word note can be associated with multiple form features at the same time". Again, De Bot introduced Green (1986)'s views on the three activations of different languages. Green (1986) argues that when a bilingual or multilingual person uses a language, a certain language is not completely open or closed, and it is highly probable that in a specific context, a certain language is selected (selected), while other languages are potentially activated. (active) or dormant (dormant).

\section{Application of Bilingual Speech Production Models and Its Development}

The bilingual speech production models can be used to explain the language output of bilinguals. In the process of communication, the two-language students use the macro plan and the micro-planning rules to screen the language. The selected part of the language is the words produced by the speaker. The model can explain the phenomenon of code switching and cross-linguistic influences in language use, and can also be used to explain the phenomenon of speech control in the process of bilingual output. In short, the bilingual speech production models are used in a wide range of applications. Therefore, more and more scholars pay attention to and promote the development of the bilingual speech production models. Kormos (2006) argues that the bilingual speech production models is modular and consists of discrete coding modules: the concept shaper, the discourse generator, and the sounder, each of which handles a specific input. Similar to the native language output, the L2 output can also be incrementally work, that is, the fragmentary input of the module can trigger the coding process of the module. For example, once the first syllable of a word is phonologically encoded, the utterer begins its pronunciation, which means that learners who achieve a certain level of language can perform parallel processing. However, as long as a coding program requires conscious attention control, its encoding can only be serial. However, this bilingual speech production models is not a serial model in the strict sense because cascading is allowed to flow from the vocabulary to the phoneme layer. In other words, a word node that is activated but not selected can continue activation to the underlying phoneme node. In addition, the model does not allow for active backward flow between levels, and its monitoring is done through a language understanding system. Kormos' bilingual speech production models also explore the unique issues of the bilingual language output process: language selection, bilingual vocabulary, syntactic coding of second language, phoneme encoding, and monitoring issues. He believes that there is no significant difference between the bilingual speech production models and the monolingual model, except for the new knowledge storage and the integration of the twolanguage concept, the word, the vocabulary and the phonetic rules that increase the declarative knowledge of the output rules. The results of neuroimaging research on bilingual output also support the view of basic similarities in the processing of mother tongue and two languages. Compared with the previous bilingual language output model, Kormos's model is more elaborate and more targeted, and highlights the difference between bilingual language output and native language.

\section{The Problem and Deficiency of Bilingual Speech Production Models}

De Bot's bilingual speech production models points out the homogeneity between the bilingual speech production models and the mother-tongue output model. Although the model is concise and vivid, it provides a good theoretical basis and method for bilingual output, but its shortcomings. The point is that the difference between the second language 
and the native language output is not clear enough. Therefore, the model still needs to be studied and improved. Kormos's bilingual speech production models are more detailed than De Bot's bilingual speech production models. This not only results in the development of the bilingual speech production models theory, but also involves problems unique to the second language output process: language selection, bilingual lexicon, and two Syntax coding, phoneme encoding, and monitoring issues. Although Kormos is concerned with the problems unique to the second language output, he did not propose a detailed and effective solution. Therefore, the bilingual model theory still needs further research and development by researchers in related fields.

\section{CONCLUSION}

Syntactic priming has become a method of studying syntactic representation and production mechanism from the perspective of psycholinguistics. This linguistic phenomenon has begun to receive more and more attention from psycholinguists. Research methods related to syntactic priming also provide an experimental paradigm for studying sentence generation, and also provide a channel for studying the importance of syntactic representation and process. The syntactic priming phenomenon explains the intricate relationship in the process of syntactic representation. Semantic priming allows researchers to understand the lexical semantic representation. Compared with the semantic start-up, syntactic priming has gained a lot of research, not only explaining the process of syntactic representation and sentence production, but also helping to reveal the mechanism of sentence comprehension.

In addition, there is still a lot of research space for syntactic priming. Recent studies have shown that the syntactic activation in Broca's aphasia indicates that the priming effect is still strong when the language is severely weakened. This indicates that even if the patient with aphasia cannot convert the internal knowledge into the spoken language, their underlying language representation remains intact.

And can form sentence concepts normally. It shows that syntactic priming is an automatic and inherent process. There is also an experimental study of the priming effect of syntax in children's verbal dialogues, thus demonstrating that children also use special structural representations in speech production. There are also people who suggest that language proficient language learners are not affected by syntax priming because they use more syntactic details in their communication. These issues need to be further studied in order to establish a more general meaning of speech production theory.

This paper summarizes the bilingual speech production models of De Bot and Kormos by using Levelt's mother tongue output model theory as an introduction, and expresses his views on the problems and shortcomings of the research. Due to the complexity of bilingual output and the lack of relevant literature, the research on bilingual speech production models theory has a long way to go. This study shows that the study of bilingual output makes us understand the nature of bilingualism and pay more attention to language. The relationship with cognition, in the process of communication, will choose the corresponding code according to the specific situation and more freely convert the two. In short, the existing research results provide a reliable theoretical basis for us to understand the output of the second language.

\section{REFERENCES}

[1] Bock, J. K.\& R. K. Conceptual accessibility and syntactic structure in sentence formulation. [J] Cognition,1985, 21:47-67.

[2] Bock. K. Sound, and syntax: Lexical priming in sentence production. [J] Journal of Experimental Psychology: Learning, Memory, and Cognition, 1986a,12: 575-586.

[3] Bock, JK. Syntactic persistence in language production[J] Cognitive psychology, 1986b, 18:355-387

[4] Bock, JK, Close Class immanence in sentence production [ J] .Cognition, 1989, 31:163 -186

[5] Green, D. Control, activation and resource. Brain and Language, 1986. 27, 210-223.

[6] Kormos, J. Speech Production and Second Language Acquisition. [M] New Jersey: Lawrence Erlbaum Associates. 2006.

[7] Levelt, W. J. M. Speaking: From Intention to Articulation. [M] Cambridge, MA: MIT Press. 1989

[8] Levelt, W. J. M., Roelofs, A. \& Meyer, A. S. A theory of lexical access in speech production. [J] Behavioral and Brain Sciences, 1999.22, 1-75.

[9] Potter, M.C, Lombardi, L. Syntactic priming in immediate recall of sentences [ J] Journal of Memory and Language, 1998(38): 265-282

[10] Pickering, M.J, Branigan, H.P. The representation of verbs: evidence from syntactic priming in language production[ J] . Journal of Memory and Language1998:633-651

[11] Pickering, M.J, Branigan, H.P. Syntactic priming in language production[J] Rrends in cognitive science, 1999(3):136 - 141.

[12] Yang Jie, ZhangYaxu. Syntactic priming in language production [J] , Progress in psychological science, 2007, 15(2):288 -294 